Benoît P. Guery

Maiken C. Arendrup

Georg Auzinger

Élie Azoulay

Márcio Borges Sá

Elizabeth M. Johnson

Eckhard Müller

Christian Putensen

Coleman Rotstein

Gabriele Sganga

Mario Venditti

Rafael Zaragoza Crespo

Bart Jan Kullberg

Received: 22 April 2008

Accepted: 5 October 2008

Published online: 30 October 2008

(C) The Author(s) 2008. This article is published with open access at Springerlink.com

Part II is published at:

doi:10.1007/s00134-008-1339-6.

B. P. Guery

Infectious Diseases, SGRIVI,

Hopital Huriez, CHRU Lille,

59045 Lille Cedex, France

M. C. Arendrup

Unit of Mycology,

Department of Bacteriology,

Mycology and Parasitology,

Statens Serum Institut, Building 43/117,

2300 Copenhagen, Denmark

\section{G. Auzinger}

Liver Intensive Care,

Institute of Liver Studies,

King's College Hospital, Denmark Hill,

London SE5 9RS, UK

É. Azoulay

Service de Réanimation Médicale,

Hôpital Saint-Louis, 1, Avenue Claude-

Bellefaux, 75010 Paris, France

M. Borges Sá

Sepsis Unit, Intensive Care Department, Hospital Son Llàtzer, Palma de Mallorca, Spain

\title{
Management of invasive candidiasis and candidemia in adult non-neutropenic intensive care unit patients: Part I. Epidemiology and diagnosis
}

E. M. Johnson

Mycology Reference Laboratory,

National Collection of Pathogenic Fungi,

The HPA Centre for Infections,

HPA South West Laboratory,

Myrtle Road, Kingsdown,

Bristol BS2 8EL, UK

E. Müller

Universitätsklinik für Anästhesiologie,

Intensivmedizin und Schmerztherapie,

Knappschaftskrankenhaus Bochum,

Langendreer, Klinikum der Ruhr,

Universität, In der Schornau 23-25,

44892 Bochum, Germany

\section{Putensen}

Operative Intensivmedizin,

Klinik und Poliklinik für Anästhesiologie und Operative Intensivmedizin,

Rheinische-Friedrich-Wilhelms Universität

Bonn, Sigmund-Freud Str. 25, 53127 Bonn,

Germany

C. Rotstein

Division of Infectious Diseases,

University Health Network,

Toronto General Hospital, NCSB 11-1212,

200 Elizabeth Street, Toronto, ON,

M5G 2C4, Canada

\section{G. Sganga}

Division of General Surgery and Organ

Transplantation,

Policlinico 'A Gemelli',

Department of Surgery, Catholic University, Largo Gemelli, 8, 00168 Rome, Italy

M. Venditti

Internal Medicine,

Clinical Medicine Department,

"La Sapienza" University, Rome,

Viale dell' Università, 37, 00185 Rome, Italy
R. Zaragoza Crespo

Secretario GTEI-SEMICYUC,

Medicina Intensiva Hospital Universitario

Dr. Peset, Avenida Gaspa Aguilar, 90,

46107 Valencia, Spain

\section{B. J. Kullberg (ه)}

Department of Medicine (463),

Nijmegen Institute for Infection,

Inflammation, and Immunity (N4i),

Radboud University Nijmegen Medical

Centre, PO Box 9101, 6500 HB Nijmegen,

The Netherlands

e-mail: b.kullberg@aig.umcn.nl

Tel.: +31-24-3668015

Fax: +31-24-3541734

Abstract Background: Invasive candidiasis and candidemia are frequently encountered in the nosocomial setting, particularly in the intensive care unit (ICU). Objectives and methods: To review the current management of invasive candidiasis and candidemia in non-neutropenic adult ICU patients based on a review of the literature and a European expert panel discussion. Results and conclusions: Candida albicans remains the most frequently isolated fungal species followed by $C$. glabrata. The diagnosis of invasive candidiasis involves both clinical and laboratory parameters, but neither of these are specific. One of the main features in diagnosis is the evaluation of risk factor for infection which will identify patients in need of pre-emptive or empiric treatment. Clinical scores were built from those risk 
factors. Among laboratory diagnosis, a positive blood culture from a normally sterile site provides positive evidence. Surrogate markers have also been proposed like 1,3 $\beta$-D glucan level, mannans, or PCR testing. Invasive candidiasis and candidemia is a growing concern in the ICU, apart from cases with positive blood cultures or fluid/tissue biopsy, diagnosis is neither sensitive nor specific. The diagnosis remains difficult and is usually based on the evaluation of risk factors.

Keywords Antifungal - Azole . Candida $\cdot$ Candidiasis .
Echinocandins · Invasive candidiasis $\cdot \mathrm{PaO}_{2}$ Intensive care Polyenes

\begin{tabular}{|c|c|c|}
\hline \multicolumn{2}{|c|}{ Abbreviations } & SBP \\
\hline aPTT & $\begin{array}{l}\text { Activated partial } \\
\text { thromboplastin time }\end{array}$ & SDD \\
\hline CAGT & Candida albicans germ tube & \\
\hline CI & Colonization index & \\
\hline DLY & Discounted life year & \\
\hline $\begin{array}{l}\mathrm{FIO}_{2} \\
\mathrm{HwP1}\end{array}$ & Fraction of inspired oxygen & \\
\hline ICU & Intensive care unit & \\
\hline INR & $\begin{array}{l}\text { International normalized } \\
\text { ratio }\end{array}$ & \\
\hline $\mathrm{AP}$ & Mean arterial blood pressure & \\
\hline & Odds ratio & \\
\hline
\end{tabular}

Partial pressure of arterial oxygen

Polymerize chain reaction Systolic blood pressure

Standard deviation

Selective digestive decontamination

Surgical intensive care unit Systemic inflammatory response syndrome

Mixed venous oxygen saturation White blood cell

\section{Introduction}

Among documented invasive fungal infections, candidemia and invasive candidiasis are encountered with increasing incidence in nosocomial settings [1]. These infections cause considerable morbidity and mortality. Several studies have estimated that $6-11 \%$ of all positive nosocomial bloodstream infections could be attributed to Candida spp. [1, 2]. However, a recent US study showed that between 1991 and 2003, the mortality rate associated with invasive candidiasis decreased slightly over time [2]. C. albicans has remained the main pathogen overall, although the frequency of $C$. glabrata increases with age.

Data from a US surveillance study found that over a 7year period Candida spp. accounted for 4.6 bloodstream infections per 10,000 admissions and 9\% of all bloodstream infections [1], whereas the incidence may vary between centers. The estimated incidence of candidemia also varies between countries In Europe, Denmark has reported the highest incidence with 11.0 cases/100,000/ year compared with a study carried out in Finland which only recorded 1.9 cases/100,000/year in the hospital population [3-5]. Candida spp. are generally reported to be the fourth-most prevalent pathogen isolated in blood cultures or deep-site infections although this prevalence varies depending on the population surveyed $[1,2]$. In intensive care units (ICUs), a slightly higher incidence is usually observed; in one study carried out in the ICU setting, candidemia accounted for $10.1 \%$ of blood stream infections compared with $7.9 \%$ on the general ward [1] Luzzati et al. [6] reported a study showing that candidemia occurred more frequently in ICUs than on either surgical or general medical wards. The infection rates were cited as 15.8/10,000 patient-days in ICUs versus $0.15 / 10,000$ on medical wards and $0.69 / 10,000$ patientdays on surgical wards. Bougnoux et al. [7] observed a mean incidence of candidemia of 6.7/1,000 admissions in ICU patients in France, and candidemia occurred more frequently in non-neutropenic patients than in patients with neutropenia [1,7]. Invasive candidiasis and candidemia are associated with increased ICU and hospital stay of 12.7 and 15.5 days, respectively, and increased total costs $[8-10]$.

Overall, ICU candidiasis represent one-third of all invasive candidiasis and is associated with a high mortality rate [11]. A recent study carried out in an adult ICU in France showed a $61.8 \%$ crude mortality [7]. A perhaps more clinically relevant parameter is the attributable mortality. This parameter estimates the excess of mortality attributable to the fungal infection compared with the mortality rate in patients matched for underlying disease and other risk factors. Thus, attributable mortality may estimate how mortality may be decreased by effective antifungal therapy. Attributable mortality of candidiasis was evaluated retrospectively between 1997 and 2001 in 108 matched pairs [12], the crude mortality among case patients was $61 \%$ compared with $12 \%$ in controls; the resulting "attributable mortality" was therefore estimated to be $49 \%$. A study performed in the US evaluating candidemia associated with septic shock and multiple organ failure showed that, although relatively infrequent in the non-immunocompromized patient, it was associated with a very high mortality rate [13]. Although not statistically significant, the mortality rate at 28 days in this study was $60 \%$ in candidemic septic shock patients, compared with $46 \%$ in bacteremic septic shock patients. Falagas et al. [14] also assessed the impact of candidemia on hospital mortality in a systematic review of seven matched cohort and case-control studies. The mortality attributed to candidemia, in the reviewed studies, ranged from 5 to $71 \%$, and for six, the difference in mortality between cases and controls was statistically 
significant. The authors concluded that despite the methodological heterogeneity, these data suggest that candidemia is associated with considerable mortality. In another study recently published, Tumbarello et al. [15] retrospectively studied the risk factors for mortality of patients with candidemia. The multivariate analysis identified three factors associated with mortality: inadequate antifungal therapy, infection with biofilm-forming Candida species, and Apache III score.

The increasing incidence of non-albicans Candida species could be important, as a prospective study in a medical-surgical ICU suggested that candidemia due to non-albicans species was associated with higher mortality [16]. Blot et al. [17] compared critically ill patients with fungemia due to C. albicans and C. glabrata. They found that patients infected by $C$. glabrata were significantly older and showed a trend toward a higher mortality. In cancer patients, Viscoli et al. [18] also suggested that $C$. glabrata was associated with a higher mortality rate. Comparing fluconazole-susceptible with resistant strains in 161 patients, Kovacicova et al. [19] found a significantly higher attributable mortality in patients infected with a fluconazole-resistant strain. On the contrary, crude mortality was not different between patients infected by $C$. glabrata or C. albicans (respectively 41 vs. $44 \%, P=0.7$ ) in a recent case control study [20], suggesting that uncertainty exists around the relative mortality of different Candida species, requiring well-controlled studies.

Despite the overwhelming evidence identifying the increasing mortality and morbidity burden of invasive candidiasis and candidemia, and its adverse impact on morbidity and mortality of critically-ill patients, the optimal management, even in the high-risk ICU patient, is still debated in the medical literature.

The aim of this article is to summarize the current management of invasive candidiasis and candidemia in adult non-neutropenic ICU patients based on a review of the international literature.

\section{Candida spp. epidemiology in the intensive care unit}

Only a limited number of studies have specifically focused on Candida spp. encountered in the ICU. In one study carried out over a 4-year period, Aliyu et al. [21] investigated 92 episodes in 90 patients, $C$. albicans was the most-frequently isolated fungal species, $C$. glabrata was second. All isolated Candida spp. were susceptible to amphotericin B, and only $87 \%$ were susceptible to fluconazole.

A larger study conducted over a 5-year period in Italy recorded 182 episodes of ICU candidemia, with an average incidence of 2.22 episodes/10,000 patient-days/ year [22]. The authors observed an increased incidence of candidemia over the years: overall, $40 \%$ of cases were due to C. albicans followed by C. parapsilosis (23\%), C. glabrata (15\%), C. tropicalis (9\%), and other species $(13 \%)$. The results of this study reflected a shift toward an increased rate of infection with non-albicans Candida species. This observation correlated with the increasing use of azoles for prophylaxis or empirical treatment, which will be discussed later in this review although this finding has not been corroborated by Shorr et al. in a recently published study [23]. The influence of azole prophylaxis on Candida epidemiology has not been clearly elucidated yet.

A hospital-based study conducted in England and Wales reported 18.7 episodes of candidemia/100,000 finished consultant episodes, $45.4 \%$ of which occurred in the ICU. C. albicans was isolated in $64.7 \%$ of confirmed cases [24].

In a large European study, Tortorano et al. [25] showed that Candida albicans was responsible for more than half of the cases in all patient populations. Candida glabrata was the most frequent non-albicans isolate in surgical (16\%) patients. These authors concluded that there was a limited role of species with decreased susceptibility to azoles in causing bloodstream infections and a low proportion of antifungal resistance.

In a study from Turkey, 302 isolates from 270 ICU patients were collected from various samples; $C$. albicans was the most frequent species detected $(65.6 \%)$ followed by C. parapsilosis (11.3\%) and C. glabrata (8.8\%) [26]. Of all the isolates, $92.9 \%$ were susceptible to fluconazole. In Canada, 409 Candida isolates were recovered during a 1-day point-prevalence study in 35 ICUs [27]. C. albicans accounted for $72 \%$ of the isolated species, followed by $C$. glabrata (16\%). Only $4 \%$ of the isolates were resistant to fluconazole and/or itraconazole.

\section{Diagnosis of invasive candidiasis and candidemia}

The diagnosis of candidiasis is still a major challenge in the ICU, and it is often made late in the course of the infection. This can be explained by several factors: clinical manifestations are non-specific, blood cultures are usually not positive until late in the course of infection, and, in approximately $50 \%$ of patients, blood culture sample size may be inadequate, i.e., not performed according to guidelines with a sample size $\geq 20 \mathrm{~mL}$ of blood [28, 29]. Finally, serological tests and cultures, apart from blood cultures, are non-specific and their diagnostic accuracy is still debated [29-31]; as a result, clinicians often disregard a potential diagnosis of candidiasis. An additional diagnostic hurdle relates to the fact that ICU patients may have received prophylactic doses of fluconazole (e.g., $100 \mathrm{mg}$ ) which may render samples negative at the time of testing. 
A diagnosis usually requires clinical, microbiological, and biochemical evidence of infection, which even if positive may not be sufficient or specific enough to guide optimal treatment. One of the main features in diagnosis is the evaluation of risk factors for infection. Possible risk factors have been evaluated in several studies and used to identify patients in need of pre-emptive or empiric treatment $[32,33]$. The optimal timing of these therapeutic options has still not been completely elucidated. Most frequently this latter objective was achieved by calculating a clinical risk score derived from identification of predetermined risk factors in patients suspected of having a fungal infection. It is appropriate to describe these risk factors first before commenting on clinically relevant risk scores. The specific role of colonization will then be discussed before assessing the relevance of biological tests.

\section{Assessment of risk factors}

Commonly recognized risk factors for invasive Candida infection are listed in Table 1 [2, 34, 35]. Several authors have used multivariate analyses in an attempt to assess independent risk factors associated with invasive candidiasis [28, 32, 33]. Using a case control study, Wey et al. [36] identified four factors associated with high risk of candidiasis: number of antibiotics received prior to infection (odds ratio (OR), 1.73 per unit increase); isolation of Candida spp. from sites other than blood (OR, 10.37); previous hemodialysis (OR, 18.13), and prior use of a Hickman catheter (OR, 7.23). Possible risk factors must be analyzed with extreme caution as they also depend on the study population: in one study, surgical ICU (SICU) patients with severe acute pancreatitis who did develop invasive candidiasis could not be differentiated from those who did not become infected when evaluated according to classical parameters such as Apache II score or previous antibiotic treatment [37]. In another study focusing on Candida peritonitis in a SICU, four variables could be identified: the Apache II score, respiratory failure on

Table 1 Commonly recognized risk factors for invasive Candida infection [2, 34, 35]

Risk factors

Neutropenia

Cancer chemotherapy

Colonization with Candida spp.

Broad-spectrum antibiotic use

Presence of a central venous catheter

Hemodialysis or renal failure

Severity of illness (Apache score)

Parenteral nutrition

Mechanical ventilation

Prior surgery

Age admission, upper gastrointestinal tract site peritonitis, and positive results for Candida following direct testing of peritoneal fluid [38]. These observations were confirmed in a recent study in 59 consecutive multidisciplinary ICU patients where both high colonization index and recent extensive gastro-abdominal surgery were correlated with invasive candidiasis and candidemia [39]. Other risk factors have also been identified, such as the presence of a central venous catheter [21] or hemodialysis [40]. In a multicenter study on risk factors in surgical patients, the incidence of fungal infections increased from $0.98 / 1,000$ to $1.42 / 1,000$ SICU days when a central venous catheter was in place [34]. Another major factor associated with an increased risk of invasive candidemia is the length of stay in the ICU; in a small study, Pelz et al. [41] showed a clear increase in risk beyond the seventh day of stay.

\section{Prediction rules}

In an attempt to improve this risk factor driven approach, several authors have tried to develop models to identify independent factors that are predictive of invasive candidiasis, and use these factors to build clinically relevant scores that may help clinicians to identify, implement and adapt an optimal therapeutic approach. In a two-stage study, Michalopoulos et al. [42] identified independent predictive factors and prospectively validated them in two centers. Independent predictors were ongoing invasive mechanical ventilation $\geq 10$ days, hospital-acquired bacterial infection and/or bacterium, cardiopulmonary bypass duration $>120 \mathrm{~min}$, and diabetes mellitus. Of these, the first two factors were the strongest predictors. This study needs, however, to be analyzed cautiously because it only involved 19 patients with candidemia. In another study, Leon et al. [28] described a clinical score based on four parameters derived from a logit model: surgery, multifocal colonization, total parenteral nutrition, and severe sepsis. A cut-off value of 2.5 was associated with a sensitivity of $81 \%$ and a specificity of $74 \%$. Using a less formal approach, Ostrosky-Zeichner et al. attempted to identify patients at high risk for invasive candidiasis in the ICU. The bestperforming rule was $\geq 1$ day of systemic antibiotic therapy or presence of a central venous catheter, and at least two of the following: total parenteral nutrition, any form of dialysis, any major surgery, pancreatitis, any use of steroids, or use of an immunosuppressive agent [33].

\section{Clinical diagnostic criteria}

Of all the risk factors discussed in the previous section, Candida colonization should be highlighted. Invasive Candida infections represent a growing challenge in the ICU and as a consequence treatment of high-risk patients is more frequently initiated pre-emptively or empirically. 
One of the main concerns in the ICU, therefore, is how to identify these patients and propose appropriate treatment algorithms, with the presumption that, in most cases, the risk of invasive candidiasis and candidemia is related to the density and extent of fungal colonization over time. One of the most important questions, which if answered positively would support the rationale for prophylaxis, is whether colonizing species portend subsequent fungal infection. An earlier study by Petri et al. [43] showed that $64 \%$ of ICU patients were colonized, and that all infected patients had been previously colonized. A related question as yet unanswered is whether colonization of certain sites carries more predictive impact versus other colonization sites.

Fungal burden was also found to be an independent risk factor in a multivariate analysis carried out on predictors of fungal infections found in ICU patients [41]. More recent studies using microsatellite markers have confirmed that, in most cases, the fungal acquisition was mainly endogenous $[44,45]$.

In a pioneer study, Pittet et al. [46] proposed a clinical colonization index to assess fungal colonization in highrisk SICU patients. In this 6-month prospective cohort study, the investigators evaluated 29 patients. Of these, 11 (38\%) developed severe infections (8 candidemia); the others were heavily colonized but did not require specific therapy. The results of this study identified two independent factors that predicted subsequent invasive Candida infection: the severity of illness as assessed by the Apache II score, and the intensity of Candida spp. colonization defined as the colonization index (CI). In this study the colonization index was defined as the number of distinct non-blood body sites (dbs) colonized by Candida spp. over the total number of distinct sites tested per patient. The results of this study led the authors to conclude that systematic screening of critically ill patients with risk factors had the potential to identify those requiring so-called pre-emptive therapy with the threshold for intervention set at a CI of 0.5. The authors developed a corrected index (product of the CI times the ratio of the number of dbs showing heavy growth to the total of dbs growing Candida spp.) which was associated with a $100 \%$ sensitivity and specificity.

Laboratory diagnosis of invasive candidiasis and candidemia

As mentioned previously, diagnosis of invasive candidiasis and candidemia remains a great challenge, since symptoms and signs are usually non-specific, microbiological cultures are difficult to analyze, and histological specimens require invasive procedures $[29,30,46]$.

A positive blood culture or the isolation of Candida spp. from a normally sterile site (except urine) provides test results that are easy to analyze, but all too often this level of positive evidence is not available to the clinician. Several techniques have recently been proposed to assist the clinician and improve the diagnostic accuracy.

\section{Surrogate markers}

$(1 \rightarrow 3)$ - $\beta$-D-glucan. ( $(1 \rightarrow 3)$ - $\beta$-D-glucan is a component of the cell wall of many fungi and has been proposed as a non-specific marker for invasive fungal infections. Using commercially available assays (colorimetric or kinetic), this method was evaluated to add another element to the diagnostic panel for invasive candidiasis. Sensitivity and specificity have been estimated to be 69.9 and $87.1 \%$, respectively [47], as there are a high number of false positive results. Furthermore the specificity of the test is hampered by ß-glucan contamination of certain antibiotics and materials, such as surgical gauzes, requiring further validation of the assay in the appropriate ICU setting before routine use can be recommended.

Mannans and other markers. Like glucans, mannans are major components of the $C$. albicans cell wall, but in contrast to glucans, mannans are non-covalently bound at the cell surface and are highly immunogenic [48]. The use of mannan antigenemia has been suggested to facilitate the diagnosis of invasive candidiasis; the most important limitation was rapid clearance of the antigen from the patient's sera [49]. To improve test performance, Sendid et al. suggested combining antigen and antibody detection. This possibility was explored using 162 serum samples selected from 63 patients with clinically proven candidiasis, compared with 98 control samples [50]. Combined analysis showed a sensitivity and specificity of 80 and 93\%, respectively, suggesting a potential value in clinical practice. This test was also effective with non-albicans Candida species [51]. A second test based on the detection of betalinked oligomannoses was subsequently developed and associated with the analysis of alpha-linked oligomannoses. The results showed a slight improvement in specificity to $95 \%$, with a sensitivity of $90 \%$ [52]. The routine use of these tests could be valuable to increase early diagnosis, but does not, by itself, offer a definitive solution for diagnosis.

Other tests have also been evaluated. A $C$. albicans germ tube antibody (CAGTA) detection test was evaluated and compared to a standard test in a retrospective study [53]. Using 172 sera from 51 hematological and intensive care patients, Candida albicans IFA IgG test showed a sensitivity of $84.4 \%$ and a specificity of $94.7 \%$, while the standard test showed a sensitivity of $78.1 \%$ and a specificity of $100 \%$. Several other antigens expressed on the $C$. albicans cell wall have been recently identified. Specific antibodies directed toward the hyphal wall protein 1 (Hwp1) were developed and compared with CAGT antibodies [54]. Detection of these antigens needs additional clinical confirmation. 
Polymerase chain reaction. The amplification of genomic sequences through polymerase chain reaction (PCR) testing has mostly been developed for invasive aspergillosis but is not routinely used in invasive candidiasis. A recent Japanese publication describes a novel PCR assay directed to five common Candida spp. [55]. A recent study evaluated prospectively, in non neutropenic ICU patients, three TaqMan-based polymerase chain reaction assays and the results showed a 90.9\% sensitivity, and $100 \%$ specificity suggesting a potential usefulness of this method [56]. These results need further evaluation.

\section{Conclusion}

ICU patients have many risk factors for developing invasive candidiasis or candidemia. For the specialist, the management of invasive candidiasis and candidemia, from diagnosis to selection of the therapeutic protocol, is often a challenge. Apart from cases with positive blood cultures or fluid/tissue biopsy, diagnosis is neither sensitive nor specific. It relies on many different factors including clinical and laboratory findings, but there is clearly a need for more specific diagnostic markers.

Conflict of interest Benoit Guery was consultant for GSK, Wyeth, and Pfizer. Maiken C Arendrup received honoraries as a speaker from Astellas, MSD, Swedish Orphan, Pfizer, Schering Plough and Cephalon and a research grant from Pfizer, and Schering Plough. Georg Auzinger received honoraria from Pfizer for advisory activities. Elie Azoulay has received research grants from Pfizer France and honorarium for lectures from Pfizer and Gilead. Marcio Borges Sá received honoraries as a speaker from MSD, Cephalon, Pfizer, Astra-Zeneca and Wyeth. Elisabeth Johnson received speakers honoraria from Gilead, MSD, Pfizer and Schering Plough, consultant honoraria from Gilead, MSD, Pfizer and ScheringPlough and research funding from Gilead. Eckhard Müller was speaker and received consultation fees for Astra Zeneca, SanofiAventis Germany, Bayer Vital, Biosyn Arzneimittel, Biotest AG, Fresenius Medical Care, MSD Sharp \& Dohme, Novartis Pharma, Pfizer Pharma, Wyeth Pharma and participated to Advisory Boards of Caspofungin (Fa. MSD Sharp \& Dohme GmbH; Merck, USA), Voriconazol (Fa. Pfizer Pharma GmbH), Anidulafungin (Fa. Pfizer, Germany; Fa. Pfizer, Europa), and Posaconazol (Fa. Essex Pharma). Coleman Rotstein received Grants/Research Supports from Astellas, Basilea, Johnson \& Johnson, Merck, Pfizer, Wyeth; was consultant for Astellas, Bayer, Merck, Pfizer, Wyeth and belonged to the speakers Bureau of Bayer, Merck, Pfizer, Wyeth. Gabriele Sganga received honoraries as a speaker from Pfizer, Gilead and Wyeth. Mario Venditti received honoraries as a speaker from Angelini, Aventis, Bayer, Glaxo, Novartis, Pfizer, Wyeth and as a consultant from Novartis and Wyeth. Bart Jan Kullberg was consultant for Basilea, Novartis, and Pfizer. The other authors do not declare any conflict of interest.

Open Access This article is distributed under the terms of the Creative Commons Attribution Noncommercial License which permits any noncommercial use, distribution, and reproduction in any medium, provided the original author(s) and source are credited.

\section{References}

1. Wisplinghoff $\mathrm{H}$, Bischoff $\mathrm{T}$, Tallent SM, Seifert H, Wenzel RP, Edmond MB (2004) Nosocomial bloodstream infections in US hospitals: analysis of 24,179 cases from a prospective nationwide surveillance study. Clin Infect Dis 39:309-317

2. Pfaller MA, Diekema DJ (2007) Epidemiology of invasive candidiasis: a persistent public health problem. Clin Microbiol Rev 20:133-163

3. Arendrup MC, Fuursted K, GahrnHansen B, Jensen IM, Knudsen JD, Lundgren B, Schonheyder HC, Tvede M (2005) Seminational surveillance of fungemia in Denmark: notably high rates of fungemia and numbers of isolates with reduced azole susceptibility. J Clin Microbiol 43:4434-4440

4. Arendrup MC, Fuursted K, GahrnHansen B, Schonheyder HC, Knudsen JD, Jensen IM, Bruun B, Christensen JJ, Johansen HK (2008) Semi-national surveillance of fungaemia in Denmark 2004-2006: increasing incidence of fungaemia and numbers of isolates with reduced azole susceptibility. Clin Microbiol Infect 14:487-494
5. Poikonen E, Lyytikainen O, Anttila VJ, Ruutu P (2003) Candidemia in Finland, 1995-1999. Emerg Infect Dis 9:985990

6. Luzzati R, Allegranzi B, Antozzi L, Masala L, Pegoraro E, Azzini A. Concia E (2005) Secular trends in nosocomial candidaemia in nonneutropenic patients in an Italian tertiary hospital. Clin Microbiol Infect 11:908-913

7. Bougnoux ME, Kac G, Aegerter P, d'Enfert C, Fagon JY (2008) Candidemia and candiduria in critically ill patients admitted to intensive care units in France: incidence, molecular diversity, management and outcome. Intensive Care Med 34:292-299

8. Wey SB, Mori M, Pfaller MA, Woolson RF, Wenzel RP (1988) Hospitalacquired candidemia. The attributable mortality and excess length of stay. Arch Intern Med 148:2642-2645

9. Rentz AM, Halpern MT, Bowden R (1998) The impact of candidemia on length of hospital stay, outcome, and overall cost of illness. Clin Infect Dis 27:781-788
10. Olaechea PM, Palomar M, Leon-Gil C, Alvarez-Lerma F, Jorda R, Nolla-Salas J, Leon-Regidor MA (2004) Economic impact of Candida colonization and Candida infection in the critically ill patient. Eur J Clin Microbiol Infect Dis 23:323-330

11. Marchetti O, Bille J, Fluckiger U, Eggimann P, Ruef C, Garbino J, Calandra T, Glauser MP, Tauber MG, Pittet D (2004) Epidemiology of candidemia in Swiss tertiary care hospitals: secular trends, 1991-2000. Clin Infect Dis 38:311-320

12. Gudlaugsson O, Gillespie S, Lee K, Vande BJ, Hu J, Messer S, Herwaldt L, Pfaller M, Diekema D (2003) Attributable mortality of nosocomial candidemia, revisited. Clin Infect Dis 37:1172-1177

13. Hadley S, Lee WW, Ruthazer R, Nasraway SA Jr (2002) Candidemia as a cause of septic shock and multiple organ failure in nonimmunocompromised patients. Crit Care Med 30:1808-1814 
14. Falagas ME, Apostolou KE, Pappas VD (2006) Attributable mortality of candidemia: a systematic review of matched cohort and case-control studies. Eur J Clin Microbiol Infect Dis 25:419-425

15. Tumbarello M, Posteraro B, Trecarichi EM, Fiori B, Rossi M, Porta R, de Gaetano DK, La Sorda M, Spanu T, Fadda G, Cauda R, Sanguinetti M (2007) Biofilm production by Candida species and inadequate antifungal therapy as predictors of mortality for patients with candidemia. J Clin Microbiol 45:1843-1850

16. Dimopoulos G, Ntziora F, Rachiotis G, Armaganidis A, Falagas ME (2008) Candida albicans versus non-albicans intensive care unit-acquired bloodstream infections: differences in risk factors and outcome. Anesth Analg 106:523-529

17. Blot S, Vandewoude K, Hoste E, Poelaert J, Colardyn F (2001) Outcome in critically ill patients with candidal fungaemia: Candida albicans vs. Candida glabrata. J Hosp Infect 47:308-313

18. Viscoli C, Girmenia C, Marinus A, Collette L, Martino P, Vandercam B, Doyen C, Lebeau B, Spence D, Krcmery V, De Pauw B, Meunier F (1999) Candidemia in cancer patients: a prospective, multicenter surveillance study by the Invasive Fungal Infection Group (IFIG) of the European Organization for Research and Treatment of Cancer (EORTC). Clin Infect Dis 28:1071-1079

19. Kovacicova G, Krupova Y, Lovaszova M, Roidova A, Trupl J, Liskova A, Hanzen J, Milosovic P, Lamosova M, Macekova L, Szovenyiova Z, Purgelova A, Obertik T, Bille J, Krcmery V (2000) Antifungal susceptibility of 262 bloodstream yeast isolates from a mixed cancer and non-cancer patient population: is there a correlation between in-vitro resistance to fluconazole and the outcome of fungemia? J Infect Chemother 6:216221

20. Klevay MJ, Ernst EJ, Hollanbaugh JL, Miller JG, Pfaller MA, Diekema DJ (2008) Therapy and outcome of Candida glabrata versus Candida albicans bloodstream infection. Diag Microbiol Infect Dis 60:273-277

21. Aliyu SH, Enoch DA, Abubakar II, Ali R, Carmichael AJ, Farrington M, Lever AM (2006) Candidaemia in a large teaching hospital: a clinical audit. QJM 99:655-663

22. Bassetti M, Righi E, Costa A, Fasce R, Molinari MP, Rosso R, Pallavicini FB, Viscoli C (2006) Epidemiological trends in nosocomial candidemia in intensive care. BMC Infect Dis 6:21
23. Shorr AF, Lazarus DR, Sherner JH, Jackson WL, Morrel M, Fraser VJ, Kollef MH (2007) Do clinical features allow for accurate prediction of fungal pathogenesis in bloodstream infections? Potential implications of the increasing prevalence of non-albicans candidemia. Crit Care Med 35:1077-1083

24. Kibbler CC, Seaton S, Barnes RA, Gransden WR, Holliman RE, Johnson EM, Perry JD, Sullivan DJ, Wilson JA (2003) Management and outcome of bloodstream infections due to Candida species in England and Wales. J Hosp Infect 54:18-24

25. Tortorano AM, Peman J, Bernhardt H, Klingspor L, Kibbler CC, Faure O, Biraghi E, Canton E, Zimmermann K, Seaton S, Grillot R (2004) Epidemiology of candidaemia in Europe: results of 28-month European Confederation of Medical Mycology (ECMM) hospital-based surveillance study. Eur J Clin Microbiol Infect Dis 23:317-322

26. Comert F, Kulah C, Aktas E, Eroglu O, Ozlu N (2007) Identification of Candida species isolated from patients in intensive care unit and in vitro susceptibility to fluconazole for a 3-year period. Mycoses 50:52-57

27. Laverdiere M, Labbe AC, Restieri C, Rotstein C, Heyland D, Madger S, Stewart T (2007) Susceptibility patterns of Candida species recovered from Canadian intensive care units. J Crit Care 22:245-250

28. Leon C, Ruiz-Santana S, Saavedra P, Almirante B, Nolla-Salas J, AlvarezLerma F, Garnacho-Montero J, Leon MA (2006) A bedside scoring system ("Candida score") for early antifungal treatment in nonneutropenic critically ill patients with Candida colonization. Crit Care Med 34:730-737

29. Fluckiger U, Marchetti O, Bille J, Eggimann P, Zimmerli S, Imhof A, Garbino J, Ruef C, Pittet D, Tauber M, Glauser M, Calandra T (2006) Treatment options of invasive fungal infections in adults. Swiss Med Wkly 136:447-463

30. Morrell M, Fraser VJ, Kollef MH (2005) Delaying the empiric treatment of candida bloodstream infection until positive blood culture results are obtained: a potential risk factor for hospital mortality. Antimicrob Agents Chemother 49:3640-3645

31. Spellberg BJ, Filler SG, Edwards JE Jr (2006) Current treatment strategies for disseminated candidiasis. Clin Infect Dis 42:244-251
32. Paphitou NI, Ostrosky-Zeichner L, Rex JH (2005) Rules for identifying patients at increased risk for candidal infections in the surgical intensive care unit: approach to developing practical criteria for systematic use in antifungal prophylaxis trials. Med Mycol 43:235243

33. Ostrosky-Zeichner L, Sable C, Sobel J, Alexander BD, Donowitz G, Kan V, Kauffman CA, Kett D, Larsen RA, Morrison V, Nucci M, Pappas PG, Bradley ME, Major S, Zimmer L, Wallace D, Dismukes WE, Rex JH (2007) Multicenter retrospective development and validation of a clinical prediction rule for nosocomial invasive candidiasis in the intensive care setting. Eur J Clin Microbiol Infect Dis 26:271276

34. Blumberg HM, Jarvis WR, Soucie JM, Edwards JE, Patterson JE, Pfaller MA Rangel-Frausto MS, Rinaldi MG, Saiman L, Wiblin RT, Wenzel RP (2001) Risk factors for candidal bloodstream infections in surgical intensive care unit patients: the NEMIS prospective multicenter study. The National Epidemiology of Mycosis Survey. Clin Infect Dis 33:177-186

35. Sobel JD, Rex JH (2001) Invasive candidiasis: turning risk into a practical prevention policy? Clin Infect Dis 33:187-190

36. Wey SB, Mori M, Pfaller MA, Woolson RF, Wenzel RP (1989) Risk factors for hospital-acquired candidemia. A matched case-control study. Arch Intern Med 149:2349-2353

37. De Waele JJ, Vogelaers D, Blot S, Colardyn F (2003) Fungal infections in patients with severe acute pancreatitis and the use of prophylactic therapy. Clin Infect Dis 37:208-213

38. Dupont H, Paugam-Burtz C, MullerSerieys C, Fierobe L, Chosidow D, Marmuse JP, Mantz J, Desmonts JM (2002) Predictive factors of mortality due to polymicrobial peritonitis with Candida isolation in peritoneal fluid in critically ill patients. Arch Surg 137:1341-1346

39. Agvald-Ohman C, Klingspor L, Hjelmqvist H, Edlund C (2007) Invasive candidiasis in long-term patients at a multidisciplinary intensive care unit: Candida colonization index, risk factors, treatment and outcome. Scand J Infect Dis 40:145-153

40. Munoz P, Burillo A, Bouza E (2000) Criteria used when initiating antifungal therapy against Candida spp. in the intensive care unit. Int J Antimicrob Agents 15:83-90 
41. Pelz RK, Lipsett PA, Swoboda SM, Diener-West M, Hammond JM, Hendrix CW (2000) The diagnostic value of fungal surveillance cultures in critically ill patients. Surg Infect (Larchmt) 1:273-281

42. Michalopoulos AS, Geroulanos S, Mentzelopoulos SD (2003)

Determinants of candidemia and candidemia-related death in cardiothoracic ICU patients. Chest 124:2244-2255

43. Petri MG, Konig J, Moecke HP, Gramm HJ, Barkow H, Kujath P, Dennhart R, Schafer H, Meyer N, Kalmar P, Thulig P, Muller J, Lode H (1997)

Epidemiology of invasive mycosis in ICU patients: a prospective multicenter study in 435 non-neutropenic patients. Paul-Ehrlich Society for Chemotherapy, Divisions of Mycology and Pneumonia Research. Intensive Care Med 23:317325

44. Stephan F, Bah MS, Desterke C, Rezaiguia-Delclaux S, Foulet F, Duvaldestin P, Bretagne S (2002) Molecular diversity and routes of colonization of Candida albicans in a surgical intensive care unit, as studied using microsatellite markers. Clin Infect Dis 35:1477-1483

45. Eloy O, Marque S, Botterel F, Stephan F, Costa JM, Lasserre V, Bretagne S (2006) Uniform distribution of three Candida albicans microsatellite markers in two French ICU populations supports a lack of nosocomial crosscontamination. BMC Infect Dis 6:162

46. Pittet D, Monod M, Suter PM, Frenk E, Auckenthaler R (1994) Candida colonization and subsequent infections in critically ill surgical patients. Ann Surg 220:751-758
47. Ostrosky-Zeichner L, Alexander BD, Kett DH, Vazquez J, Pappas PG, Saeki F, Ketchum PA, Wingard J, Schiff R, Tamura H, Finkelman MA, Rex JH (2005) Multicenter clinical evaluation of the (1->3) beta-D-glucan assay as an aid to diagnosis of fungal infections in humans. Clin Infect Dis 41:654-659

48. Fukazawa Y (1989) Antigenic structure of Candida albicans. Immunochemical basis of the serologic specificity of the mannans in yeasts. Immunol Ser 47:3762

49. Poulain D, Robert R, Mesnard F, Sendid B, Lepage G, Camus D (1997) Clearances of Candida albicansderived alpha- and beta-linked mannose residues in sera from patients with candidiasis. Eur J Clin Microbiol Infect Dis 16:16-20

50. Sendid B, Tabouret M, Poirot JL, Mathieu D, Fruit J, Poulain D (1999) New enzyme immunoassays for sensitive detection of circulating Candida albicans mannan and antimannan antibodies: useful combined test for diagnosis of systemic candidiasis. J Clin Microbiol 37:15101517

51. Sendid B, Poirot JL, Tabouret M, Bonnin A, Caillot D, Camus D, Poulain D (2002) Combined detection of mannanaemia and antimannan antibodies as a strategy for the diagnosis of systemic infection caused by pathogenic Candida species. J Med Microbiol 51:433-442

52. Sendid B, Jouault T, Coudriau R, Camus D, Odds F, Tabouret M, Poulain D (2004) Increased sensitivity of mannanemia detection tests by joint detection of alpha- and beta-linked oligomannosides during experimental and human systemic candidiasis. J Clin Microbiol 42:164-171
53. Moragues MD, Ortiz N, Iruretagoyena JR, Garcia-Ruiz JC, Amutio E, Rojas A, Mendoza J, Quindos G, Ponton-San Emeterio J (2004) Evaluation of a new commercial test (Candida albicans IFA $\mathrm{IgG}$ ) for the serodiagnosis of invasive candidiasis. Enferm Infecc Microbiol Clin 22:83-88

54. Lain A, Elguezabal N, Brena S, GarciaRuiz JC, Del Palacio A, Moragues MD, Ponton J (2007) Diagnosis of invasive candidiasis by enzyme-linked immunosorbent assay using the $\mathrm{N}$-terminal fragment of Candida albicans hyphal wall protein 1 . BMC Microbiol 7:35

55. Arishima T, Takezawa J (2006) Use of PCR based diagnosis for common invasive fungal infections in the intensive care unit. Nippon Ishinkin Gakkai Zasshi 47:283-288

56. McMullan R, Metwally L, Coyle PV, Hedderwick S, McCloskey B, O'Neill HJ, Patterson CC, Thompson G, Webb CH, Hay RJ (2008) A prospective clinical trial of a real-time polymerase chain reaction assay for the diagnosis of candidemia in nonneutropenic, critically ill adults. Clin Infect Dis 46:890-896 